\title{
Trace element deficiency in freshwater cladoceran Daphnia magna
}

\author{
Isaac K. S. Lam, Wen-Xiong Wang* \\ Department of Biology, Hong Kong University of Science and Technology (HKUST), Clear Water Bay, Kowloon, \\ Hong Kong, SAR China
}

\begin{abstract}
We conducted $\mathrm{Cu}, \mathrm{Se}$, and Zn deficiency-recovery experiments and measured a suite of biological parameters such as reproduction, mortality, morphology, and dietary assimilation in the freshwater cladoceran Daphnia magna. Whether Cd could substitute for Zn or Cu when the latter metals were deficient was also tested in this animal system. Significant reduction in reproduction was observed for the first generation $\left(\mathrm{F}_{0}\right)$ of the $\mathrm{Cu}$ - or $\mathrm{Zn}$-deficient daphnids, whereas Se deficiency only caused significant reduction in reproduction in the second generation $\left(\mathrm{F}_{1}\right)$. A complete recovery of reproduction in $F_{1}$ was observed when daphnids were transferred to Se- and Cu-sufficient conditions, but only partial recovery was found for Zn. There was no significant mortality for the Se- and Zndeficient daphnids in $\mathrm{F}_{0}$, but significant mortality was observed for the $\mathrm{F}_{1}$ Se-deficient and the $\mathrm{F}_{0} \mathrm{Cu}-$ deficient daphnids. No morphological defects were observed in the metal-deficient daphnids of $F_{0}$. However, Se-deficient $F_{1}$ showed bi-phased morphological defects of the cuticle and the second antenna. Cd could not substitute $\mathrm{Cu}$ or $\mathrm{Zn}$ in the $\mathrm{D}$. magna biological requirements, but caused additional toxicity to the daphnids. A high dietary Zn assimilation efficiency was found in the Zn-deficient daphnids, whereas dietary Se assimilation was comparable between the Se-deficient and Se-sufficient daphnids, suggesting that daphnids possessed a stronger homeostatic ability for Zn than for Se. Our study demonstrated that daphnids responded differently to deficiencies of different essential trace elements, which was unlikely to have been controlled by their biokinetics (i.e. dietary assimilation).
\end{abstract}

KEY WORDS: Daphnia magna · Deficiency · Cadmium · Copper · Selenium · Zinc

Resale or republication not permitted without written consent of the publisher

\section{INTRODUCTION}

For most essential elements or metals, an optimal concentration range is required for a steady development and reproduction in organisms (Van Assche et al. 1997). Within the optimal range, organisms can regulate their internal element concentration by homeostasis. However, the homeostatic balance may fail when the concentration is out of the range. An elevated concentration can cause toxicity, and conversely, organisms may experience chemical deficiency when the concentration is lower than the optimal range. Both 'deficiency' or 'surplus' can have adverse effects on organisms; therefore, the study of both toxicity and deficiency is of equal interest. Over the past decades, the aquatic toxicity of essential and non-essential metals to aquatic animals has been well examined, but published works on metal deficiency are relatively rare. This is in contrast to the many studies of trace element limitation in marine phytoplankton (e.g. Fe and Zn limitations; Sunda et al. 1991, Morel et al. 1994, Sunda \& Huntsman 1997). A few previous studies investigated the physiological effects of Se and Zn deprivation in multiple generations of Daphnia pulex (Keating \& Dagbusan 1984, Caffrey \& Keating 1997). Keating \& Dagbusan (1984) suggested that $1 \mu \mathrm{g} \mathrm{Se} \mathrm{l}^{-1}$ was sufficient to satisfy the minimal needs of daphnids. Keating \& Caffrey (1989) examined the interaction between Se and Zn during Zn deficiency in D. magna, highlighting a higher Se demand during $\mathrm{Zn}$ deficiency). More recently, some studies have demonstrated the possibility of $\mathrm{Zn}$ and $\mathrm{Cu}$ deficiency in D. magna during or before chronic toxicity tests (Muyssen \& Janssen 2001, Bossuyt \& Janssen 2004, 
De Schamphelaere \& Janssen 2004). These studies indicated that the optimal concentrations were 300 to $600 \mu \mathrm{g} \mathrm{Zn} \mathrm{l}^{-1}$ and 1 to $35 \mu \mathrm{g} \mathrm{Cu} \mathrm{l^{-1 }}$ (Muyssen \& Janssen 2002, Bossuyt \& Janssen 2003).

During metal-deficient conditions, other metals with similar properties may substitute the deficient one(s) and perform (some of) its functions. A typical example is the substitution of $\mathrm{Zn}$ and Co with $\mathrm{Cd}$. Zhang et al. (1996) demonstrated that Cd was able to functionally substitute for Zn to activate the farnesyl:protein transferase (FPT; a recombinant rat enzyme purified from the baculovirus cell expression system). Lane \& Morel (2000) found that addition of Cd to Zn-limited cultures enhanced the growth of a marine diatom, Thalassiosira weissflogii, suggesting that $\mathrm{Cd}$ can replace $\mathrm{Zn}$ in the enzymatic functions in the carbon-concentrating mechanism. Substitution of toxic metals such as Cd for essential metals such as $\mathrm{Zn}$ has not been found in animal systems. Therefore, it is relevant to examine whether Cd can substitute Zn or other essential metals such as $\mathrm{Cu}$ during deficiency conditions in freshwater organisms.

In this study, we conducted $\mathrm{Cu}, \mathrm{Se}$, and $\mathrm{Zn}$ deficiency experiments in a freshwater cladoceran, Daphnia magna. D. magna neonates less than $24 \mathrm{~h}$ old were cultured in different metal-deficient conditions and compared to daphnids grown under sufficient (normal) conditions. Physiological parameters such as reproduction, mortality, and morphology were monitored. Deficiency-recovery (hereafter 'recovery') experiments were further carried out to investigate the recovery of daphnids from the metal deficiency conditions. We also tested whether Cd can substitute Zn or $\mathrm{Cu}$ during deficiencies of those metals. Furthermore, dietary assimilation was quantified for the first time in Se- and Zn-deficient daphnids to examine the kinetic responses to metal-deficient conditions.

\section{MATERIALS AND METHODS}

Trace metal cleaning technique. To maintain a metal deficient environment throughout this study, a trace metal cleaning technique was essential. Conical flasks for algal culture and plastic beakers for rearing the daphnids were soaked with $5 \% \mathrm{HNO}_{3}$ for $2 \mathrm{~d}$ and were rinsed thoroughly by ultra-pure water. The containers were sealed with plastic film before use. Disposable $3 \mathrm{ml}$ plastic pipettes were used for transferring the daphnids throughout the experiment. The chemicals used were analytical grade or above to minimize the possible metal contamination.

Organisms and artificial medium. The freshwater cladoceran Daphnia magna has been cultured in our laboratory for more than $6 \mathrm{yr}$, in Whatman ${ }^{\circledR}$ glass fiber
(GF/C) filtered pond water with animal to water ratio of $1 \mathrm{ind}$. to $10 \mathrm{ml}$ water at a room temperature of $23.5^{\circ} \mathrm{C}$ with a 14:10 h light:dark cycle. They were fed daily with the unicellular green alga Chlamydomonas reinhardtii at a food concentration of $10^{5} \mathrm{cell} \mathrm{ml}^{-1}$, and the water was renewed every $2 \mathrm{~d}$. The algae were maintained in an artificial WC medium (Guillard 1975), which did not contain Se as nutrient, for freshwater phytoplankton in ultra-pure water at $23.5^{\circ} \mathrm{C}$ with a 14:10 h light:dark cycle.

In this experiment, artificial medium was prepared by using Elendt M7 medium (Samel et al. 1999). We modified the M7 medium recipe by using $0 \mu \mathrm{g}$ Se $\mathrm{l}^{-1}$

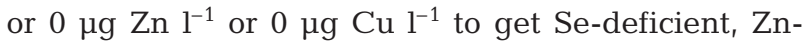
deficient and $\mathrm{Cu}$-deficient artificial media, respec-

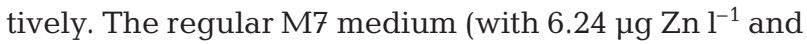
$1.57 \mu \mathrm{g} \mathrm{Cu} \mathrm{l}^{-1}$ ) was used as Zn-sufficient and Cu-sufficient artificial medium. The Se concentration listed in the $\mathrm{M} 7$ recipe was modified to $1 \mu \mathrm{g} \mathrm{Se} \mathrm{l}^{-1}$ for constructing Se-sufficient artificial medium, as $1 \mu \mathrm{g} \mathrm{Se} \mathrm{l}^{-1}$ is sufficient for daphnid minimal needs (Keating \& Dagbusan 1984). $\mathrm{Cd}\left(\mathrm{CdCl}_{2}\right)$ was used in the recovery experiment, at a nominal concentration of $1 \mu \mathrm{g} \mathrm{Cd} \mathrm{l}^{-1}$.

Reproduction, survivorship and morphology. The effects of Se deficiency on the survivorship, reproduction and morphology were examined in 2 consecutive generations of Daphnia magna. Twenty D. magna neonates less than $24 \mathrm{~h}$ old were collected (as first generation, $F_{0}$ ) from normal daphnid stock culture and transferred to GF/C filtered Se-deficient and Se-sufficient medium, separately, at a density of 0.1 ind. $\mathrm{ml}^{-1}$. There were triplicates for each treatment. The daphnids in both treatments were fed with Se-free algae at $5 \times 10^{4}$ cells ml ${ }^{-1}$ every day and the water was renewed every $2 \mathrm{~d}$. The exposure lasted for $25 \mathrm{~d}$, during which any neonates (first appearing on Day 8) and dead daphnids were discarded after counting, except for the neonates produced on Day 20 (retained as second generation, $F_{1}$ ). After counting, 10 individuals of $F_{1}$ neonates with triplicates were exposed under the same condition as their parents and 10 individuals of the Se-deficient $F_{1}$ with triplicates were exposed to Sesufficient medium (at $1 \mu \mathrm{g} \mathrm{Se}{ }^{-1}$ ) as a Se-recovery treatment, with the food supply at $5 \times 10^{4}$ cells $\mathrm{ml}^{-1}$. Survivorship and reproduction of the $F_{1}$ generation were monitored every day for $30 \mathrm{~d}$. During the exposure period, any abnormality in the tested daphnids was recorded; both normal and abnormal daphnids were photographed using a Leica DMR research microscope with 100× magnification. After the test, the daphnids were collected using a GF/C membrane for dry weight (DW) measurement and then digested using $25 \% \mathrm{HNO}_{3}$ for Se body burden measurement using the PerkinElmer AAnalyst ${ }^{\mathrm{TM}} 800$ high-performance atomic absorption spectrometer graphite furnace technique. 
The effects of $\mathrm{Zn}$ and $\mathrm{Cu}$ deficiency on the survivorship, reproduction and morphology were examined during one generational development. Forty-five Daphnia magna neonates less than $24 \mathrm{~h}$ old were collected from normal daphnid stock culture and transferred to GF/C filtered metal (Zn or $\mathrm{Cu}$ )-deficient or -sufficient medium and fed with metal ( $\mathrm{Zn}$ or $\mathrm{Cu}$ )-deficient or -sufficient algae at $5 \times 10^{4}$ cells $\mathrm{ml}^{-1}$ every day, respectively. There were triplicates for each treatment. Znand $\mathrm{Cu}$-deficient food were obtained by growing the Chlamydomonas reinhardtii in WC medium which did not contain the corresponding metal, and C. reinhardtii grown in regular WC medium was collected as the Znand $\mathrm{Cu}$-sufficient food. Other experimental conditions were similar to the Se-deficiency experiments. The total exposure lasted for $24 \mathrm{~d}$ in $\mathrm{Zn}$ treatments and $30 \mathrm{~d}$ in $\mathrm{Cu}$ treatments. Midway through the exposures, the remaining Zn-deficient daphnids at Day 12 and $\mathrm{Cu}$-deficient daphnids at Day 15 were transferred to new treatments for the recovery test. They were divided among 3 treatments: deficient, sufficient (i.e. recovery of $\mathrm{Zn}$ and $\mathrm{Cu}$ ),

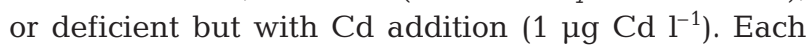
treatment had triplicates with 10 to 15 individuals per replicate. The recovery treatment was fed-metal sufficient algae, whereas the Cd treatment was fed metal-deficient algae. The $\mathrm{Zn}$-sufficient and $\mathrm{Cu}$-sufficient treatments continued to be exposed to sufficient conditions. During the whole 24 to $30 \mathrm{~d}$ experimental period, mortality and reproduction were carefully monitored; any neonates produced and dead daphnids were counted and then discarded. Any abnormalities in the test daphnids were recorded; both abnormal and normal daphnids were photographed using a Leica DMR research microscope with $100 \times$ magnification. After the test, the daphnids (about 15) were collected using a GF/C membrane for DW measurement and then digested using $25 \% \mathrm{HNO}_{3}$ for $\mathrm{Zn}$ and $\mathrm{Cu}$ body burden measurement using the Perkin-Elmer AAnalyst ${ }^{\mathrm{TM}} 800$ high-performance atomic absorption spectrometer graphite furnace technique.

Assimilation efficiency of Se-deficient and Zn-deficient daphnids. The radiotracer technique was used to determine the biokinetics of Se and $\mathrm{Zn}$ non-invasively in the daphnids during the experiment. Biokinetics of $\mathrm{Cu}$ were not quantified due to the unavailability of a suitable $\mathrm{Cu}$ isotope for our study. $\mathrm{H}_{2}{ }^{75} \mathrm{SeO}_{3}$ (in $0.1 \mathrm{~N}$ $\mathrm{HCl}$, half-life $t_{1 / 2}=119.78 \mathrm{~d}$, with a specific activity of 26.3 $\mathrm{GBq} \mathrm{g}^{-1}$ ) was obtained from Risø National Laboratory, Roskilde, Denmark. ${ }^{65} \mathrm{ZnCl}_{2}$ (in $0.1 \mathrm{~N} \mathrm{HCl} t_{1 / 2}=$ $244 \mathrm{~d}$, with a specific activity of $1.665 \mathrm{GBq} \mathrm{mg}^{-1}$ ) was obtained from Brookhaven National Laboratory, New York, USA. The radioactivity of isotopes was determined by a Wallac $1480 \mathrm{NaI}$ (T1) gamma counter (Turku). Counting times were adjusted to yield propagated counting errors of $<10 \%$.
The impacts of Se (first generation: $F_{0}$, and second generation: $F_{1}$ ) and $\mathrm{Zn}$ deficiency on their respective assimilation efficiencies (AEs) were quantified. To radiolabel the algae with $\mathrm{Se}$ and $\mathrm{Zn}$, log phase Chlamydomonas reinhardtii were transferred to a modified WC medium which did not contain $\mathrm{Cu}$, Zn, or EDTA (Wang et al. 1996). For the Se radiolabeling, phosphate was not added to the WC medium, because an earlier study demonstrated that phosphate greatly reduced the Se uptake by competitive interaction (Yu $\&$ Wang 2004). The initial cell density was adjusted to $10^{5}$ cells ml ${ }^{-1}$. Radioactive Se (at $263 \mathrm{kBq} \mathrm{l}^{-1}$ ) and $\mathrm{Zn}$ (at $146 \mathrm{kBq} \mathrm{l}^{-1}$ ) were added to the media at a concentration of $10 \mu \mathrm{g} \mathrm{Se} \mathrm{l}^{-1}$ and $88 \mathrm{ng} \mathrm{Zn} \mathrm{l^{-1 }}$, respectively. The daphnids' Se and Zn exposure levels were controlled at a low level in order to prevent recovery in the deficient daphnids during exposure which might have affected the metals' uptake kinetics. The dietary Se and $\mathrm{Zn}$ intake (calculated based on the radioactivity in the algae, amount of algae ingested, and the daphnid DW) were controlled at $<63 \mathrm{ng}$ Se $\mathrm{g}^{-1}$ daphnid DW and $<13$ ng $\mathrm{Zn} \mathrm{g}^{-1}$ daphnid DW. The algae were allowed to grow for 1 to $2 \mathrm{~d}$. After that, the cells (which took up 44 and $58 \%$ of the spiked Se and $\mathrm{Zn}$, respectively) were assumed to be evenly labeled, and they were collected by centrifugation at $1881 \times g$ for $10 \mathrm{~min}$ and resuspended in GF/C filtered Se-deficient or Zn-deficient artificial medium. This regime was repeated twice in order to remove the weakly bound metals (Se or $\mathrm{Zn}$ ). The final cell density was measured by using a hemocytometer.

Individuals of Daphnia magna from both conditions (deficient and sufficient) of Se $\left(F_{0}, 21\right.$ d old; $F_{1}, 10 \mathrm{~d}$ old) and $\mathrm{Zn}$ (12 d old) treatments were separately transferred to $450 \mathrm{ml} \mathrm{GF/C} \mathrm{filtered} \mathrm{Se-deficient} \mathrm{and} \mathrm{Zn-}$ deficient synthetic pond water, respectively, at a density of 0.1 ind. $\mathrm{ml}^{-1}$, without food for $2 \mathrm{~h}$ before the experiment in order to starve them. Afterwards, the daphnids in each treatment were divided into 3 groups (triplicates) with 15 individual each, and transferred to a $200 \mathrm{ml}$ plastic beaker with $150 \mathrm{ml} \mathrm{GF/C} \mathrm{filtered} \mathrm{Se-}$ deficient or Zn-deficient medium. For each treatment, the daphnids were fed with $2 \times 10^{4}$ cells $\mathrm{ml}^{-1} \mathrm{Se}$ - or $\mathrm{Zn}$ labeled algae in the dark for $15 \mathrm{~min}$. The daphnids and any egested feces produced were then collected by sieving through $300 \mu \mathrm{m}$ mesh and $20 \mu \mathrm{m}$ mesh, respectively, and were rinsed gently with GF/C filtered Se-deficient or Zn-deficient medium before the radioactivity measurements. Metal (Se or Zn) in the feces represented a small fraction $(<5 \%)$ of the metal ingested by the daphnids during the $15 \mathrm{~min}$ pulsefeeding period. The sum of metal in the daphnids and in the feces represented the total amount of metal ingested by the daphnids during the 15 min pulsefeeding period. After the radioactivity measurements, 
the daphnids were depurated in GF/C filtered Sedeficient or Zn-deficient medium with $2 \times 10^{4}$ cells ml $^{-1}$ unlabeled algae for $24 \mathrm{~h}$. The radioactivity in the daphnids was measured and the water and food were renewed every $4 \mathrm{~h}$. The water and food were renewed every $4 \mathrm{~h}$ in the first $12 \mathrm{~h}$ of the depuration. By assuming that the algae were completely digested by the daphnids within $12 \mathrm{~h}$ (Wang \& Fisher 1999, Yu \& Wang 2002b), the Se or Zn assimilation efficiency (AE) was calculated as the percentage of ingested metal (Se or $\mathrm{Zn}$ ) retained in D. magna after $12 \mathrm{~h}$ of depuration.

\section{RESULTS AND DISCUSSION}

\section{Reproduction, survivorship and morphology}

In order to verify that the experimental daphnids experienced metal-deficient conditions, the body burdens of $\mathrm{Se}, \mathrm{Zn}$ and $\mathrm{Cu}$ in the daphnids were determined. The Se body burdens were not measured in the $\mathrm{F}_{0}$ daphnids. The measured Se body burdens in the Se-sufficient and Se-deficient daphnids of $\mathrm{F}_{1}$ (in $\mathrm{ng} \mathrm{g}^{-1}$ DW) were $300 \pm 100$ and $0.5 \pm 0.1$, respectively. The Zn body burdens (in $\mu \mathrm{g} \mathrm{g}^{-1} \mathrm{DW}$ ) were $101 \pm 14,54 \pm 14$, and $85 \pm 3$ for Zn-sufficient, Zn-deficient and Znrecovery daphnids, respectively. The $\mathrm{Cu}$ body burdens (in $\mu \mathrm{g} \mathrm{g}^{-1} \mathrm{DW}$ ) were 10.6 $\pm 1.1,0.8 \pm 0.4$ and $7.2 \pm 3.3$ for $\mathrm{Cu}$-sufficient, $\mathrm{Cu}$-deficient, and $\mathrm{Cu}$-recovery daphnids, respectively. Both $\mathrm{Zn}$ - and $\mathrm{Cu}$-deficient daphnids showed a significantly lower (2 and 10 times, respectively) body burden compared with the -sufficient daphnids.

Reproduction was expressed as the total number of neonates produced per adult from the day of first brood to the day of recovery and from the day of recovery to the end of the experiment for $\mathrm{Zn}$ and $\mathrm{Cu}$ treatments. Se treatments were applied from Day 11 to 25 for both $F_{0}$ and $F_{1}$ generations (Fig. 1). Se-deficient and Se-sufficient treatments of $\mathrm{F}_{0}$ did not show significant difference in the total reproduction within the $15 \mathrm{~d}$ period (Days 11 to 25). In the $F_{1}$, however, the total reproduction of Se-deficient daphnids was significantly lower than that in the Se-sufficient and Se-recovery groups ( $p<0.05)$. Keating \& Dagbusan (1984) also found that Se-deficient daphnids had a significant decrease in reproduction in terms of brood numbers (e.g. only 6 broods in their whole lifespan, compared to 16 broods for the Se-sufficient daphnids). The recovery group did not show statistical difference with the Se-sufficient treatment in total reproduction, suggesting that the daphnids were able to fully recover during the $25 \mathrm{~d}$ period. Similar recovery has also been shown in Sedeficient rats when fed with high-Se Spirulina (75 $\mu \mathrm{g}$ Se $\mathrm{kg}^{-1}$ of diet) for $56 \mathrm{~d}$ (Cases et al. 2002).
Daphnids maintained under $\mathrm{Zn}$ - and $\mathrm{Cu}$-deficient environments showed no reproduction within the first 12 and 15 d, respectively (Fig. 1), while the Zn- and $\mathrm{Cu}$-sufficient daphnids started to reproduce on Day 8 (data not shown). During the second phase of experiments, both Zn- and Cu-sufficient daphnids had the highest total reproduction between Days 13 to 24 and Days 16 to 30, respectively. The Zn-recovery daphnids showed a significantly higher reproduction than the Zn-deficient daphnids, but did not reach the same level as the Zn-sufficient treatment, indicating that the daphnids could only partially recover from the $\mathrm{Zn}$ deficiency. The Zn-deficient daphnids had a significantly lower total reproduction within the second phase (about $60 \%$ of the Zn-sufficient treatments). Muyssen et al. (2002) found that Daphnia magna acclimated in low dissolved $\mathrm{Zn}$ conditions ( $3 \mu \mathrm{g} \mathrm{Zn} \mathrm{l}^{-1}$ ) for $21 \mathrm{~d}$ reproduced at a lower rate (10 to $50 \%$ ) compared to the con-

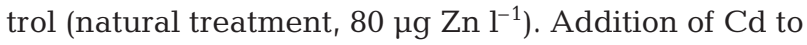
the $\mathrm{Zn}$-deficient treatment resulted in an even lower reproduction than the $\mathrm{Zn}$-deficient treatment alone, suggesting that $\mathrm{Cd}$ (at $1 \mu \mathrm{gd} \mathrm{l}^{-1}$ ) not only could not substitute $\mathrm{Zn}$ in its essential functions, but at the same time generated stress on daphnid reproduction.
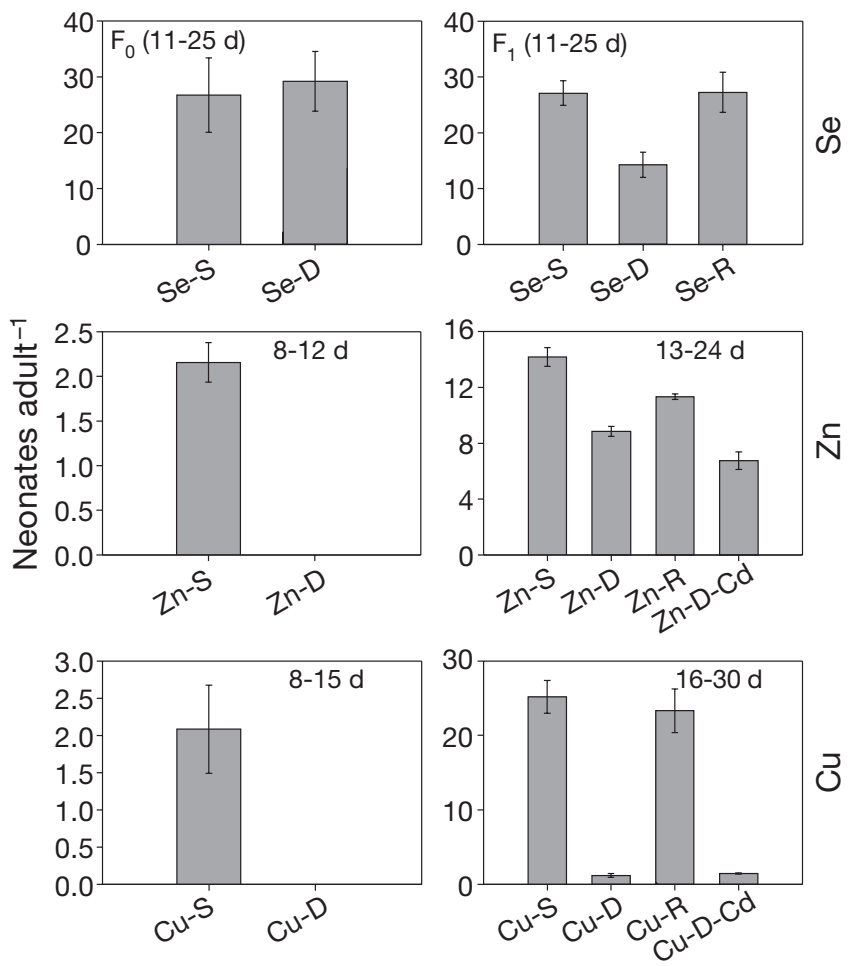

Fig. 1. Daphnia magna. Total neonates produced per adult during different experimental periods. Se experiment involved 2 generations of daphnids, whereas $\mathrm{Cu}$ and $\mathrm{Zn}$ experiments involved one single generation. S: sufficient; D: deficient; R: recovery from trace element deficiency; D-Cd: deficient with $1 \mu \mathrm{g} \mathrm{Cd} \mathrm{l}^{-1}$ added. Mean $\pm \mathrm{SD}(\mathrm{n}=3$ replicates, $10-15$ ind. per replicate) 
In contrast, the Cu-recovery treatment showed a full recovery of reproduction. In our study, the $\mathrm{Cu}-$ deficient treatment had a significantly lower reproduction, whereas De Schamphelaere \& Janssen (2004) found that the reproduction of Daphnia magna acclimated in $\mathrm{Cu}$-limited conditions for $21 \mathrm{~d}$ (with a nominal concentration of $0 \mu \mathrm{g} \mathrm{Cu} \mathrm{l}^{-1}$ ) was not significantly different from that in the Cu-sufficient treatment (35 $\mu \mathrm{g}$ $\mathrm{Cu} \mathrm{l}^{-1}$ ). One possible explanation was that the algae offered to the daphnids in that study (op. cit.) were not grown in a Cu-limited environment. Cd (at $1 \mu \mathrm{g} \mathrm{Cd} \mathrm{l}^{-1}$ ) could not substitute for $\mathrm{Cu}$ in our recovery experiment, but did not present an extra toxic effect to the reproduction of the $\mathrm{Cu}$-deficient daphnids.

Survivorship was carefully monitored throughout the experimental periods (Fig. 2). There was no mortality in the $\mathrm{F}_{0}$ daphnids in both Se-deficient and Sesufficient treatments during the $25 \mathrm{~d}$ exposure (data not shown). In the $F_{1}$ exposure, both Se-sufficient and Se-recovery treatments had an almost $100 \%$ survival throughout the exposure, whereas significant mortality was observed from Day 10 onwards for the Sedeficiency treatment. In a few replicated treatments, the mortality was as high as $100 \%$ within a $10 \mathrm{~d}$ period (Days 11 to 20). Keating \& Dagbusan (1984) also found that Se-deficient daphnids had a shortened lifespan with no progeny attaining reproductive maturity in the second generation. In our study, the $F_{1}$ could reach reproductive maturity and reproduce before they died at Days 11 to 20. Consequently, the Se-deficient condition became more severe when the daphnids reached reproductive maturity, which demanded more Se for reproduction. This may explain why the $F_{1}$ Sedeficient daphnids started to die when they reached the adult stage (11 to $12 \mathrm{~d}$ old). Muntau et al. (2002) pointed out that human Se demand is age-dependent, with later stages requiring more Se.

For Zn, all the experimental treatments (including the recovery) had high survivorship (>90\%) and did not show significant difference among the treatments, similar to results obtained by Caffrey \& Keating (1997). In the $\mathrm{Cu}$ experiments, the highest survival was found for the Cu-sufficient treatment (100\%), followed by the Cu-recovery (>90\%) and Cu-deficient treatments (87\%). Addition of $\mathrm{Cd}$ to the $\mathrm{Cu}$-deficient treatment significantly decreased the survivorship of the daphnids $(<60 \%$ at the end of the experiment), suggesting that $\mathrm{Cd}$ presented extra stress on the survivorship of the daphnids during $\mathrm{Cu}$ deficiency.

During the $25 \mathrm{~d}$ period, the morphology of the $\mathrm{F}_{0}$ Sedeficient daphnids was the same as the Se-sufficient daphnids. There were no signs of morphological defects for the $\mathrm{F}_{1}$ Se-recovery daphnids either. However, abnormalities were documented in the $F_{1}$ Sedeficient daphnids, which were differentiated into 2
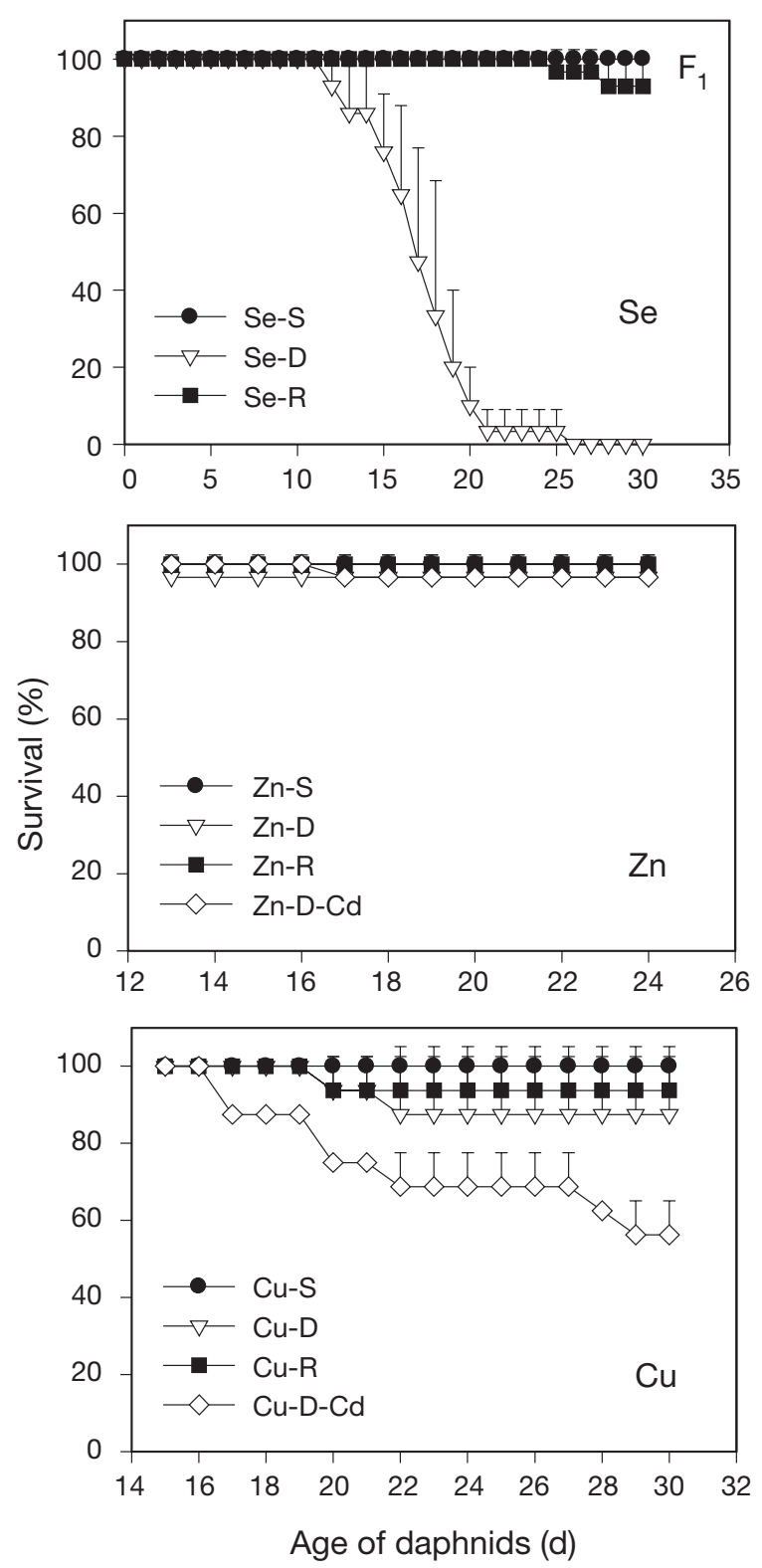

Fig. 2. Daphnia magna. Survivorship of daphnids maintained under different trace element conditions. S: sufficient; D: deficient; R: recovery from trace element deficiency; D-Cd: deficient with $1 \mu \mathrm{g} \mathrm{Cd} \mathrm{l}^{-1}$ added. Mean $\pm \mathrm{SD}(\mathrm{n}=3$ replicates, 10-15 ind. per replicate)

phases of deformation (Fig. 3). During Phase 1 (Days 9 to 10), the Se-deficient daphnids had impaired second antenna (swimming appendage), with distortion of the feather-like structure of the swimming seta, and cuticle integrity affected. During Phase 2 (Days 15 to 16), the branch structure of the second antenna totally disappeared and only the base of the antenna and the last segment of the appendage remained. At Days 15 to $16,100 \%$ of the tested daphnids had the deformation. The degeneration of the antenna (from Phase 1 to 

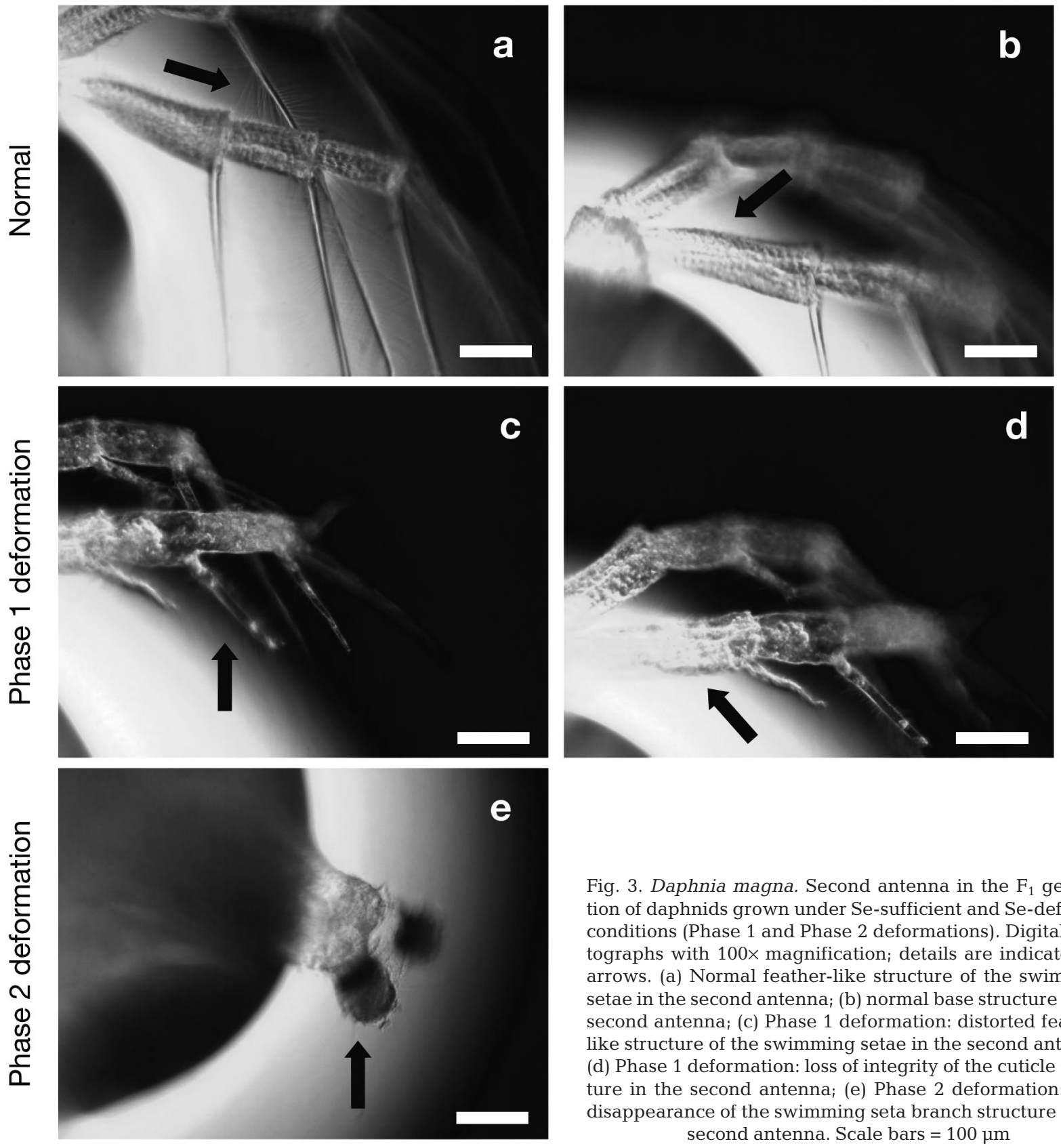

Fig. 3. Daphnia magna. Second antenna in the $F_{1}$ generation of daphnids grown under Se-sufficient and Se-deficient conditions (Phase 1 and Phase 2 deformations). Digital photographs with $100 \times$ magnification; details are indicated by arrows. (a) Normal feather-like structure of the swimming setae in the second antenna; (b) normal base structure of the second antenna; (c) Phase 1 deformation: distorted featherlike structure of the swimming setae in the second antenna; (d) Phase 1 deformation: loss of integrity of the cuticle structure in the second antenna; (e) Phase 2 deformation: total disappearance of the swimming seta branch structure in the second antenna. Scale bars $=100 \mu \mathrm{m}$

Phase 2) appeared to become progressively worse with each molt. The Phase 1 deformed daphnids were able to swim freely with a relatively vigorous motion compared to the Se-sufficient daphnids, but the Phase 2 deformed daphnids lost all mobility. The retarded mobility in the Se-deficient daphnids resulted in difficulty ingesting food particles, and they eventually died due to starvation. Similar cuticle and second antenna deformities in Daphnia pulex and D. magna were also recorded by Keating \& Dagbusan (1984), who called this process 'premature senescence' and differ- entiated the deformations into 5 stages. However, those authors found that the first morphological defect occurred in $\mathrm{F}_{0}$ after the reproduction of 10 to 12 broods. Difference in e.g. hardness (i.e. dissolved compounds) of the medium used (MS animal medium used by Keating \& Dagbusan 1984, as opposed to M7 medium used in our study) may explain the different starting times to the appearance of the defects. Se is involved in antioxidant enzymes such as glutathione peroxidase which can protect cell membranes from damage caused by the peroxidation of lipids (Tinggi 2003). The gluta- 
thione peroxidase may not function normally during Se-limited conditions and the cell membrane may be damaged by peroxidation, eventually leading to the 'premature senescence'. No morphological defects were observed in $\mathrm{Zn}$ - or $\mathrm{Cu}$-deficient daphnids in our study.

\section{Dietary assimilation of Se and Zn}

The depuration of ingested radioactive Se in Sedeficient and Se-sufficient daphnids following a pulse ingestion of food is shown in Fig. 4. There was no significant difference between the Se-deficient and Se-sufficient daphnids in both $\mathrm{F}_{0}$ and $\mathrm{F}_{1}$ generations. The calculated AEs were 57.6 and $58.5 \%$ for the $F_{0}$ Se-deficient and Se-sufficient daphnids, and were 48.9 and $53.8 \%$ for the $F_{1}$, respectively. Overall, the AEs measured for both $\mathrm{F}_{0}$ and $\mathrm{F}_{1}$ were comparable to previous measurements (33 to $58 \%$ ) of Se AEs in Daphnia magna (Yu \& Wang 2002b). These results suggested that there was no regulation of Se uptake from the dietary phase in Daphnia magna, partially because the Se demand in daphnids was low (as $1 \mu \mathrm{g} \mathrm{Se} \mathrm{l}^{-1}$ was sufficient for the minimal needs; Keating \& Dagbusan 1984). Such a low requirement of Se may not lead the daphnids to develop a regulatory mechanism for Se uptake from the dietary phase, which is the predominant route by which Se accumulates in daphnids (Canton 1999, Yu \& Wang 2002b).

Interestingly, the $\mathrm{Zn}$ retention in the $\mathrm{Zn}$-deficient daphnids was significantly higher than that of the Zn-sufficient daphnids (Fig. 4). The calculated Zn AEs in the Zn-deficient daphnids was $85.7 \%$, as compared to $53.8 \%$ in the Zn-sufficient daphnids. These data strongly implied that the daphnids may regulate the dietary Zn uptake during Zn-limited conditions. In our study, the Zn-deficient daphnids had only $66 \%$ ingestion rate of the Zn-sufficient daphnids $\left(10^{6}\right.$ cells ind. ${ }^{-1} \mathrm{~d}^{-1}$ vs. $1.5 \times 10^{6}$ cells ind. ${ }^{-1} \mathrm{~d}^{-1}$, respectively, calculated from the total amount of radioactivity in the animals after pulse feeding and the radioactivity in the algae). One possible explanation for the higher $\mathrm{Zn} \mathrm{AE}$ in the Zn-deficient daphnids was the lower ingestion rate(which may lead to a longer residence time in the gut). However, the variability of ingestion rate was not sufficient to account for the difference in Zn AEs measured between the 2 groups of daphnids. In previous studies, the typical Zn AEs in Daphnia magna were around $7 \%$ (Yu \& Wang 2002a, Guan \& Wang 2004), which were significantly lower than our current measurements for the Zn AEs in the Zn-sufficient daphnids, although the $\mathrm{Zn}$ AE quantification methods were similar. Such discrepancy may be due to the difference in experimental designs, in that our current test

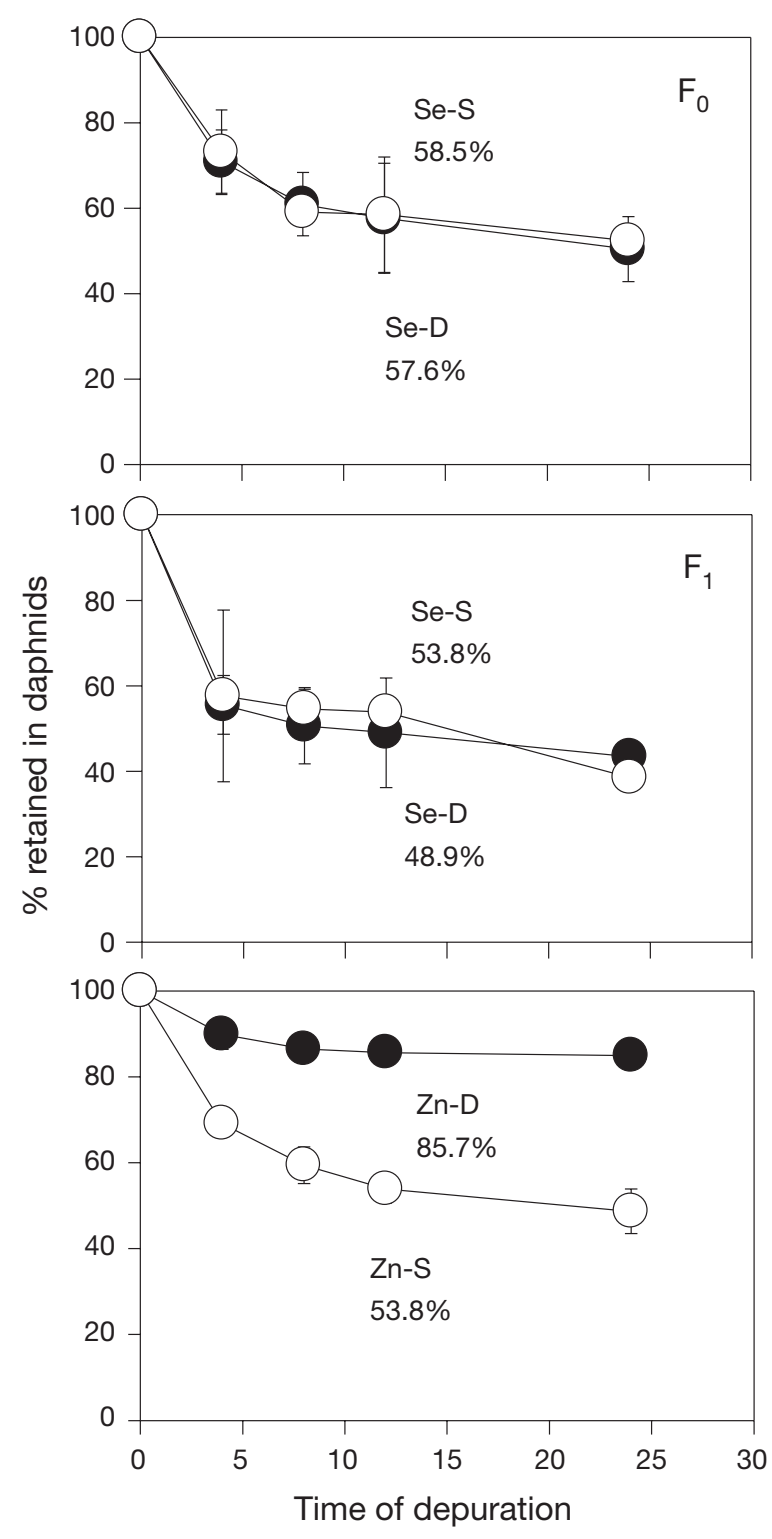

Fig. 4. Daphnia magna. Retention of ingested Se in 2 generations $\left(\mathrm{F}_{0}\right.$ and $\left.\mathrm{F}_{1}\right)$ of Se-deficient and -sufficient daphnids feeding on Chlamydomonas reinhardtii (upper panels), and retention of ingested $\mathrm{Zn}$ in $\mathrm{Zn}$-deficient and -sufficient daphnids feeding on $C$. reinhardtii (lower panel). S (open circles): sufficient; D (closed circles): deficient. Mean \pm SD $(n=3$ replicates, 10-15 ind. per replicate)

medium was Zn-deficient M7 medium and the algal food used for radioactive labeling was also grown under Zn-limited conditions. The significantly high AE measured for the Zn-deficient daphnids implies that Zn was more likely regulated during dietary uptake. Furthermore, the high AE of $\mathrm{Zn}$ in daphnids suggests that they may maximize $\mathrm{Zn}$ uptake by alternating the uptake pathways (dissolved uptake to dietary uptake) to deal with metal-limited conditions. 
In summary, daphnids responded differently to deficiencies of different trace elements. A decrease of reproduction and survivorship was observed for $\mathrm{Cu}-$ and Zn-deficient daphnids within the first generation, even though the dietary $\mathrm{Zn}$ AEs increased in Daphnia magna in response to Zn deficiency. The homeostatic regulation of dietary $\mathrm{Zn}$ uptake was not sufficient to compensate for the ambient $\mathrm{Zn}$ limitation. In contrast to $\mathrm{Cu}$ and $\mathrm{Zn}$, decrease of reproduction and survivorship was observed only for the second generation of daphnids when Se became a limiting factor. There was no apparent homeostatic regulation of Se biokinetics in response to Se limitation, and its dietary AEs remained comparable to those in the Se-sufficient environments. Major morphological defects were observed in the cuticles and the second antenna of the Se-deficient daphnids. Essential trace metal deficiency is not rare in aquatic animals, especially in artificial culture systems, such as laboratory culture and aquaculture systems. Zn and $\mathrm{Cu}$ deficiency may occur in routine laboratory culture of D. magna (Muyssen \& Janssen 2001, Bossuyt \& Janssen 2004). Consequently, there is a need to study trace metal limitation in animal systems in aquatic environments.

Acknowledgements. We gratefully acknowledge the technical assistance of Siu Chung Chi, Chi Wing Lee, and Robert Dei during the course of this work. Helpful comments by the anonymous reviewers are also much appreciated.

\section{LITERATURE CITED}

Bossuyt BTA, Janssen CR (2003) Acclimation of Daphnia magna to environmentally realistic copper concentrations. Comp Biochem Physiol C 136:253-264

Bossuyt BTA, Janssen CR (2004) Influence of multigeneration acclimation to $\mathrm{Cu}$ on tolerance, energy reserves, and homeostasis of Daphnia magna Straus. Environ Toxicol Chem 23:2029-2037

Caffrey PB, Keating KI (1997) Results of Zn deprivation in daphnid culture. Environ Toxicol Chem 16:572-575

$>$ Canton SP (1999) Acute aquatic life criteria for Se. Environ Toxicol Chem 18:1425-1432

> Cases J, Wysocka IA, Caporiccio B, Jouy N, Besançon P, Szpunar J, Rouanet JM (2002) Assessment of Se bioavailability from high-Se spirulina subfractions in Se-deficient rats. J Agric Food Chem 50:3867-3873

$>$ De Schamphelaere KAC, Janssen CR (2004) Effects of chronic dietary $\mathrm{Cu}$ exposure on growth and reproduction of Daphnia magna. Environ Toxicol Chem 23:2038-2047

Guan R, Wang WX (2004) Dietary assimilation and elimination of $\mathrm{Cd}, \mathrm{Se}$, and $\mathrm{Zn}$ by Daphnia magna at different metal concentrations. Environ Toxicol Chem 23: 2689-2698

Guillard RRL (1975) Culture of phytoplankton for feeding marine invertebrates. In: Smith WL, Chanley $\mathrm{MH}$ (eds)

Editorial responsibility: Matthias Seaman,

Oldendorf/Luhe, Germany
Culture of marine invertebrate animals. Plenum Publishing, London, p 29-60

Keating KI, Caffrey PB (1989) Se deficiency induced by Zn deprivation in a crustacean. Proc Natl Acad Sci USA 86: 6436-6440

Keating KI, Dagbusan BC (1984) Effect of Se deficiency on cuticle integrity in the Cladocera (Crustacea). Proc Natl Acad Sci USA 81:3433-3437

Lane TW, Morel FMM (2000) A biological function for Cd in marine diatoms. Proc Natl Acad Sci USA 97:4627-4631

- Morel FMM, Reinfelder JR, Roberts SB, Chamberlain CP, Lee JG, Yee D (1994) Zinc and carbon co-limitation of marine phytoplankton. Nature 369:740-742

Muntau AC, Streiter M, Kappler M, Roschinger W and others (2002) Age-related reference values for serum Se concentrations in infants and children. Clin Chem 48:555-560

Muyssen BTA, Janssen CR (2001) Multigeneration Zn acclimation and tolerance in Daphnia magna: implications for water-quality guidelines and ecological risk assessment. Environ Toxicol Chem 20:2053-2060

Muyssen BTA, Janssen CR (2002) Accumulation and regulation of zinc in Daphnia magna: links with homeostasis and toxicity. Arch Environ Contam Toxicol 43:492-496

Muyssen BTA, Janssen CR, Bossuyt BTA (2002) Tolerance and acclimation to $\mathrm{Zn}$ of field-collected Daphnia magna populations. Aquat Toxicol 56:69-79

Samel A, Ziegenfuss M, Goulden CE, Banks S, Baer KN (1999) Culturing and bioassay testing of Daphnia magna using Elendt M4, Elendt M7, and COMBO media. Ecotoxicol Environ Saf 43:103-110

Sunda WG, Huntsman SA (1997) Interrelated influence of iron, light and cell size on marine phytoplankton growth. Nature 390:389-392

Sunda WG, Swift DG, Huntsman SA (1991) Low iron requirement for growth in oceanic phytoplankton. Nature 351: 55-57

Tinggi U (2003) Essentiality and toxicity of Se and its status in Australia: a review. Toxicol Lett 137:103-110

Van Assche F, Van Tiborg W, Waeterschoot H (1997) Environmental risk assessment for essential elements, case study: Zn. In: Langley E, Mangas S (eds) Zn - report of an international meeting. National Environmental Health Forum Monographs, Metal Series No. 2, South Australian Health Commission, Adelaide, p 33-47

$>$ Wang WX, Fisher NS (1999) Assimilation efficiencies of chemical contaminants in aquatic invertebrates: a synthesis. Environ Toxicol Chem 18:2034-2045

Wang WX, Reinfelder JR, Lee BG, Fisher NS (1996) Assimilation and regeneration of trace elements by marine copepods. Limnol Oceanogr 41:70-81

Yu RQ, Wang WX (2002a) Kinetic uptake of bioavailable Cd, Se, and Zn by Daphnia magna. Environ Toxicol Chem 21: 2348-2355

Yu RQ, Wang WX (2002b) Trace metal assimilation and release budget in Daphnia magna. Limnol Oceanogr 47: 495-504

$>$ Yu RQ, Wang WX (2004) Biokinetics of Cd, Se, and Zn in freshwater alga Scenedesmus obliquus under different phosphate and nitrogen conditions and metal transfer to Daphnia magna. Environ Pollut 129:443-456

Zhang FL, Fu HW, Casey PJ, Bishop WR (1996) Substitution of $\mathrm{Cd}$ for $\mathrm{Zn}$ in farnesyl:protein transferase alters its substrate specificity. Biochemistry 35:8166-8171

Submitted: August 17, 2007; Accepted: November 29, 2007

Proofs received from author(s): January 11, 2008 